\title{
A NOTE ON FUNCTIONALLY COMPACT SPACES
}

\author{
ANGELO BELLA
}

\begin{abstract}
In this paper we give some characterizations of functionally compact Hausdorff spaces in terms of multifunctions. Also, some applications are presented.
\end{abstract}

Introduction. Hausdorff functionally compact spaces have been introduced in 1969 by Dickman and Zame [2]. Some characterizations of such spaces, in terms of covers, have been given by Goss and Viglino [5] and by Lim and Tan [8]. Other characterizations, in terms of nets, have been found by Herrington [4].

The main purpose of this note is to establish a characterization of Hausdorff functionally compact spaces in terms of multifunctions. From our main result, we then obtain some generalizations of a theorem proved by Herrington and Long in [6].

1. Preliminaries. In the sequel, we will follow the notation of [3 and 9]. We recall only some definitions.

DEFINITION 1.1. Let $X$ be a topological space. A filterbase in $X$ is a family $₹$ of subsets of $X$ with the property that for every $F_{1}, F_{2} \in \mathcal{F}$ there is $F_{3} \in \mathcal{F}$ such that $F_{3} \subseteq F_{1} \cap F_{2}$. It is allowed that $\varnothing \in \mathcal{F}$. The adherence of the filterbase $\mathcal{F}$ is the set $A=\bigcap_{F \in \mathcal{F}} \bar{F}$. We say that $\mathcal{F}$ converges to $A$ if for every open set $V \subseteq X$, with $A \subseteq V$, there exists $F \in \mathcal{F}$ such that $F \subseteq V$.

DEFINITION 1.2. Let $X, Y$ be two topological spaces. A multifunction $\Phi$ from $X$ into $Y$ (briefly, $\Phi: X \rightarrow 2^{Y}$ ) is a function from $X$ into the family of all subsets of $Y$. For every $A \subseteq X$, and $B \subseteq Y$, we put $\Phi(A)=\bigcup_{x \in A} \Phi(x)$ and $\Phi^{-1}(B)=$ $\{x \in X: \Phi(x) \cap B \neq \varnothing\}$. We say that $\Phi$ is open (resp. closed) if $\Phi(A)$ is open (resp. closed) in $Y$ for every open (resp. closed) set $A \subseteq X$. We say that $\Phi$ is upper (resp. lower) semicontinuous if $\Phi^{-1}(B)$ is closed (resp. open) in $X$ for every closed (resp. open) set $B \subseteq Y$. In particular, observe that $\Phi$ is upper semicontinuous if and only if for every $x \in X$ and every open set $V \subseteq Y$, with $\Phi(x) \subseteq V$, there exists $U \in B_{x}$ ( $B_{x}$ denotes the family of all open neighborhoods of $x$ ) such that $\Phi(U) \subseteq V$. We say that $\Phi$ is continuous if it is upper and lower semicontinuous. The graph of $\Phi$ is the set $\operatorname{Gr}(\Phi)=\{(x, y) \in X \times Y: y \in \Phi(x)\}$. We say that $\Phi$ has a closed graph if $\operatorname{Gr}(\Phi)$ is closed in $X \times Y$. It is well known that the following characterization holds:

Proposition 1.3. Let $X$ and $Y$ be two topological spaces. Then, a multifunction $\Phi: X \rightarrow 2^{Y}$ has a closed graph if and only if $\Phi(x)=\bigcap_{U \in B_{x}} \overline{\Phi(U)}$ for all $x \in X$.

DEFINITION 1.4. Let $X$ be a topological space and $A \subseteq X$. We put

$$
\operatorname{cl}_{\vartheta}(A)=\left\{x \in X: \forall U \in B_{x} \bar{U} \cap A \neq \varnothing\right\} .
$$

Received by the editors June 3, 1985 and, in revised form, October 10, 1985.

1980 Mathematics Subject Classification (1985 Revision). Primary 54D30, 54C60. 
We say that $\operatorname{cl}_{\vartheta}(A)$ is the $\vartheta$-closure of $A$ and that $A$ is $\vartheta$-closed if $A=\operatorname{cl}_{\vartheta}(A)$.

We always have $\bar{A} \subseteq \operatorname{cl}_{\vartheta}(A)$ and the equality $\bar{A}=\operatorname{cl}_{\vartheta}(A)$ holds in general only for regular spaces. In [12] an example is given of a first countable Urysohn space in which there exists a subset $A$ such that $\operatorname{card}(\bar{A})=\aleph_{0}$ and $\operatorname{card}\left(\operatorname{cl}_{\vartheta}(A)\right)=2^{\aleph_{0}}$.

DEFINITION 1.5 (see [10]). A topological space $X$ is said to be $C$-compact if for every closed set $A \subset X$ and every open cover $\mathcal{U}$ of $A$ there exist finitely many $U_{1}, \ldots, U_{n} \in \mathcal{U}$ such that $A \subseteq \bigcup_{i=1}^{n} \bar{U}_{i}$.

Proposition 1.6 (see [11, Theorem 3c]). A topological space $X$ is $C$ compact if and only if every open filterbase $\mathcal{U}$ converges to its adherence.

DEFINITION 1.7 (see [2]). A Hausdorff space $X$ is said to be functionally compact if and only if every open filterbase $\mathcal{U}$ such that $\bigcap_{U \in \mathcal{U}} U=\bigcap_{U \in \mathcal{U}} \bar{U}$ converges to its adherence.

Proposition 1.8 (see [2, Theorem 3]). A Hausdorff space $X$ is functionally compact if and only if, for any Hausdorff space $Y$, every continuous function from $X$ onto $Y$ is closed.

DEFINITION 1.9. A Hausdorff space $(X, \tau)$ is said to be minimal Hausdorff if there exists no Hausdorff topology on $X$ which is strictly weaker than $\tau$.

Proposition 1.10 (see [2, Theorem 2]). A Hausdorff space $X$ is minimal Hausdorff if and only if every open filterbase $\mathcal{U}$ for which there is $x_{0} \in X$ such that $\left\{x_{0}\right\}=\bigcap_{U \in \mathcal{U}} U=\bigcap_{U \in \mathcal{U}} \bar{U}$ converges to $\left\{x_{0}\right\}$.

In the class of Hausdorff spaces, every compact space is $C$-compact, every $C$ compact space is functionally compact, every functionally compact space is minimal Hausdorff and none of the above implications is reversible (see $[\mathbf{2}, \mathbf{5}, \mathbf{8}, \mathbf{1 0}]$ ).

2. Main results. In this section we give some characterizations of Hausdorff functionally compact spaces in terms of multifunctions. We also present some results about $C$-compact spaces and minimal Hausdorff spaces.

THEOREM 2.1. A Hausdorff space $Y$ is functionally compact if and only if, for any Hausdorff space $X$, every open multifunction $\Phi: X \rightarrow 2^{Y}$, with closed graph, is upper semicontinuous.

Proof. Necessity. Let $Y$ be functionally compact. Let $X$ and $\Phi$ be as in the statement. Fix $x \in X$. Since $\Phi$ is open, the family $\{\Phi(U)\}_{U \in B_{x}}$ is an open filterbase. Moreover, by Proposition 1.3, we have $\Phi(x)=\bigcap_{U \in B_{x}} \Phi(U)=\bigcap_{U \in B_{x}} \overline{\Phi(U)}$. From Definition 1.7, it follows that the filter $\{\varphi(U)\}_{U \in B_{x}}$ converges to $\Phi(x)$, that is for any open set $V \subseteq Y$, with $\Phi(x) \subseteq V$, there is $U \in B_{x}$ such that $\Phi(U) \subseteq V$. This proves that $\Phi$ is upper semicontinuous.

Sufficiency. By Proposition 1.8, it suffices to prove that for any Hausdorff space $X$, every continuous function $f$ from $Y$ onto $X$ is closed. Consider the multifunction $\Phi: X \rightarrow 2^{Y}$ defined by putting $\Phi(x)=f^{-1}(x)$ for all $x \in X$. Since $f$ is continuous, $\Phi$ is open and, furthermore, taking into account that $X$ is Hausdorff, we have

$$
\bigcap_{U \in B_{x}} \overline{\Phi(U)}=\bigcap_{U \in B_{x}} \overline{f^{-1}(U)} \subseteq \bigcap_{U \in B_{x}} f^{-1}(\bar{U})=f^{-1}\left(\bigcap_{U \in B_{x}} \bar{U}\right)=f^{-1}(x)=\Phi(x) .
$$


Hence, $\Phi(x)=\bigcap_{U \in B_{x}} \overline{\Phi(U)}$. Then, by Proposition 1.3, $\Phi$ is upper semicontinuous, and so $f$ is closed (see [9]).

We now present an analogous version of the preceding theorem for minimal Hausdorff spaces.

THEOREM 2.2. A Hausdorff space $Y$ is minimal Hausdorff if and only if, for any Hausdorff space $X$, every open function $f: X \rightarrow Y$, with closed graph, is continuous.

Proof. Necessity. Let $Y$ be minimal Hausdorff. Let $X$ and $f$ be as in the statement. Fix $x \in X$. Since $f$ is open, the family $\{f(U)\}_{U \in B_{x}}$ is an open filterbase. Moreover, $\{f(x)\}=\bigcap_{U \in B_{x}} f(U)=\bigcap_{U \in B_{x}} \overline{f(U)}$. Then, by Proposition 1.10, the filter $\{f(U)\}_{U \in B_{x}}$ converges to $\{f(x)\}$, that is for any open set $V \subseteq Y$, with $f(x) \in V$, there is $U \in B_{x}$ such that $f(U) \subseteq V$. This proves that $f$ is continuous.

Sufficiency. If $X$ is a Hausdorff space with the same support of $Y$ but with a weaker topology, the identity $i: X \rightarrow Y$ is open and, for all $x \in X$, we have $\{i(x)\}=\bigcap_{U \in B_{x}} \overline{i(U)}$. Then, by hypothesis, $i$ is continuous. This proves that the topology of $X$ cannot be strictly weaker than that of $Y$. Hence, $Y$ is minimal Hausdorff.

Now, let us recall the following definition.

Definition 2.3 (see [7]). A topological space $X$ is said to be weakly seminormal if each of its $\vartheta$-closed subsets has a fundamental system of regular open neighborhoods.

In [8], Lim and Tan showed that a Hausdorff space is functionally compact if and only if it is minimal Hausdorff and weakly seminormal. Thanks to this result and to Theorem 2.2, we obtain the following.

COROLlARY 2.4. A weakly seminormal Hausdorff space $Y$ is functionally compact if and only if, for any Hausdorff space $X$, every open function $f: X \rightarrow Y$, with closed graph, is continuous.

For $C$-compact spaces, a result similar to the necessary part of Theorem 2.1 holds.

THEOREM 2.5. Let $Y$ be a $C$-compact space. Then, for any topological space $X$, every open multifunction $\Phi: X \rightarrow 2^{Y}$, with closed graph, is upper semicontinuous.

ProOF. If one uses Proposition 1.6, the proof is similar to that of the necessary part of Theorem 2.1.

DEFINITION 2.6. Let $X, Y$ be two topological spaces and $\Phi: X \rightarrow 2^{Y}$. We say that $\Phi$ is strongly subclosed if $\bigcap_{U \in B_{x}} \operatorname{cl}_{\vartheta}(\Phi(U \backslash\{x\})) \subseteq \Phi(x)$ for all $x \in X$. We say that $\Phi$ is strongly closed if it is strongly subclosed and its values are $\vartheta$-closed.

REMARK. If in Definition 2.6 the space $Y$ is assumed to be regular, we can say, following other terminology, that the multifunction $\Phi$ is strongly subclosed if and only if, for every $x \in X$, the topological upper limit of $\Phi$ at $x$ is contained in $\Phi(x)$ (see [13 and 14]).

In [1], Clay and Joseph proved that a topological space $Y$ is $C$-compact if and only if, for any topological space $X$, every strongly subclosed multifunction $\Phi: X \rightarrow$ $2^{Y}$ is upper semicontinuous. We now give an analogous version of this result for functionally compact Hausdorff spaces. 
To this end, let us recall the following

Proposition 2.7 (see [8, Theorem 2.6]). A Hausdorff space is functionally compact if and only if every open filterbase, with $\vartheta$-closed adherence, converges to its adherence.

We now prove the following

THEOREM 2.8. A Hausdorff space $Y$ is functionally compact if and only if, for any Hausdorff space $X$, every strongly closed multifunction $\Phi: X \rightarrow 2^{Y}$ is upper semicontinuous.

Proof. Necessity. Let $Y$ be functionally compact. Let $X$ and $\Phi$ be as in the statement. Fix $x \in X$. We have

$$
\begin{aligned}
\Phi(x) & \subseteq \bigcap_{U \in B_{x}} \operatorname{cl}_{\vartheta}(\Phi(U))=\bigcap_{U \in B_{x}} \operatorname{cl}_{\vartheta}(\Phi(U \backslash\{x\}) \cup \Phi(x)) \\
& =\bigcap_{U \in B_{x}} \operatorname{cl}_{\vartheta}(\Phi(U \backslash\{x\})) \cup \operatorname{cl}_{\vartheta}(\Phi(x)) \\
& =\bigcap_{U \in B_{x}} \operatorname{cl}_{\vartheta}(\Phi(U \backslash\{x\})) \cup \Phi(x) .
\end{aligned}
$$

Hence, $\Phi(x)=\bigcap_{U \in B_{x}} \operatorname{cl}_{\vartheta}(\Phi(U))$. Consider the open filterbase $\mathcal{V}=\{V \subseteq Y: V$ is open and there is $U \in B_{x}$ such that $\left.\Phi(U) \subseteq V\right\}$. Let us prove that the adherence of $\mathcal{V}$ is $\Phi(x)$. Of course, $\Phi(x) \subseteq \bigcap_{V \in \mathcal{V}} \overline{\bar{V}}$. Hence, it suffices to prove that if $y \in Y \backslash \Phi(x)$, then there exists $V_{y} \in \mathcal{V}$ such that $y \notin \bar{V}_{y}$. Indeed, since $\Phi(x)=$ $\bigcap_{U \in B_{x}} \operatorname{cl}_{\vartheta}(\Phi(U))$, from the fact that $y \notin \Phi(x)$, it follows that there exists $U \in B_{x}$ such that $y \notin \operatorname{cl}_{\vartheta}(\Phi(U))$. Hence, by the definition of $\vartheta$-closure, there exists an open set $V_{y} \subseteq Y$ such that $\Phi(U) \subseteq V_{y}$ and $y \notin \bar{V}_{y}$. Since $\Phi(x)$ is $\vartheta$-closed, by Proposition 2.7, the filter $\mathcal{V}$ converges to $\Phi(x)$, and so for every open set $W \subseteq Y$, with $\Phi(x) \subseteq W$, there is $V \in \mathcal{V}$ such that $V \subseteq W$. Therefore, there exists $U \in B_{x}$ such that $\Phi(U) \subseteq W$. This proves that $\phi$ is upper semicontinuous.

Sufficiency. Thanks to the sufficient part of Theorem 2.1, it suffices to prove that, for any Hausdorff space $X$, every open multifunction $\Phi: X \rightarrow 2^{Y}$ such that $\Phi(x)=\bigcap_{U \in B_{x}} \overline{\Phi(U)}$ for all $x \in X$, is upper semicontinuous. Therefore, let $\Phi$ be such a multifunction. Since the closure of any open set coincides with the $\vartheta$-closure, we have that $\Phi(x)=\bigcap_{U \in B_{x}} \operatorname{cl}_{\vartheta}(\Phi(U))$. Moreover, $\Phi(x)$ is $\vartheta$-closed. Indeed, if $y \in Y \backslash \Phi(x)$, from the above relation, it follows that there is $U \in B_{x}$ such that $y \notin \operatorname{cl}_{\vartheta}(\Phi(U))$, and so, a fortiori, $y \notin \operatorname{cl}_{\vartheta}(\Phi(x))$. Thus, $\Phi(x)=\operatorname{cl}_{\vartheta}(\Phi(x))$. Then, by hypothesis, $\Phi$ is upper semicontinuous.

3. Applications. In [6, Theorem 11], Herrington and Long proved that if $X$ is a regular space and $Y$ is a $C$-compact space, every open and closed function $f: X \rightarrow Y$, with closed point inverses, is continuous. In this section we shall give some generalizations of this result.

THEOREM 3.1. Let $X$ be a topological space and $Y$ be a $C$-compact space. Then, every open and closed multifunction $\Phi: X \rightarrow 2^{Y}$, with $\vartheta$-closed point inverses, is upper semicontinuous.

ProOF. Let us prove that $\Phi(x)=\bigcap_{U \in B_{x}} \overline{\Phi(U)}$ for all $x \in U$. Since, of course, $\Phi(x) \subseteq \bigcap_{U \in B_{x}} \overline{\Phi(U)}$, it suffices to prove that if $y \in Y \backslash \Phi(x)$, then there exists $U \in$ 
$B_{x}$ such that $y \notin \overline{\Phi(U)}$. Therefore, let $y \in Y \backslash \Phi(x)$. This implies that $x \notin \Phi^{-1}(y)$. Since $\Phi^{-1}(y)$ is $\vartheta$-closed, there exists $U \in B_{x}$ such that $\bar{U} \cap \Phi^{-1}(y)=\varnothing$, and so $y \notin \Phi(\bar{U})$. Since $\Phi$ is closed, we have $\overline{\Phi(U)} \subseteq \Phi(\bar{U})$, and so, a fortiori, $y \notin \overline{\Phi(U)}$. Finally, since $\Phi$ is open, by Theorem 2.5, it is also upper semicontinuous.

THEOREM 3.2. Let $X$ be a Hausdorff space and $Y$ be a functionally compact Hausdorff space. Then, every open and closed multifunction $\Phi: X \rightarrow 2^{Y}$, with $\vartheta$-closed point inverses, is upper semicontinuous.

PROOF. By using Theorem 2.1, the proof is analogous to that of Theorem 3.1.

THEOREM 3.3. Let $X$ be a Hausdorff space and $Y$ be a minimal Hausdorff space. Then, every open and closed function $f: X \rightarrow Y$, with $\vartheta$-closed point inverses, is continuous.

ProOF. By using Theorem 2.2, the proof is analogous to that of Theorem 3.1.

Clearly, if, in the above three theorems, we assume that $X$ is regular, then it suffices to suppose that the point inverses of the multifunctions or functions considered are closed.

From Theorem 3.2 we can derive the following generalization of Proposition 1.7.

THEOREM 3.4. A Hausdorff space $X$ is functionally compact if and only if, for any Hausdorff space $Y$, every continuous multifunction $\Phi$ from $X$ onto $Y$, with $\vartheta$-closed values, is closed.

ProOF. Necessity. Let $X$ be functionally compact. Let $Y$ and $\Phi$ be as in the statement. Let $\Psi(y)=\Phi^{-1}(y)$ for all $y \in Y$. Thus, the multifunction $\Psi$ is open and closed, and its point inverses are $\vartheta$-closed. Then, by Theorem $3.2, \Psi$ is upper semicontinuous, and so $\Phi$ is closed.

Sufficiency. Let $Y$ be any Hausdorff space and $f$ be any continuous function from $X$ onto $Y$. Thus, since $Y$ is Hausdorff, the values of $f$ are $\vartheta$-closed. Therefore, by hypothesis, $f$ is closed. Hence, by Proposition $1.8, X$ is functionally compact.

\section{REFERENCES}

1. J. Clay and J. Joseph, A characterization of $C$-compact spaces, Proc. Amer. Math. Soc. 82 (1981), 657-658.

2. R. F. Dickman and A. Zame, Functionally compact spaces, Pacific J. Math. 31 (1969), 303-311.

3. R. Engelking, General topology, PWN, Warsaw, 1977.

4. L. L. Herrington, Characterizations of functionally compact spaces, J. Austral. Math. Soc. Ser. A 29 (1980), 71-79.

5. G. Goss and G. Viglino, Some topological properties weaker than compactness, Pacific J. Math. 35 (1970), 635-638.

6. L. L. Herrington and P. E. Long, Characterizations of C-compact spaces, Proc. Amer. Math. Soc. 52 (1975), 417-426.

7. T. C. Lominal, Separation properties and $C$-compact spaces, Notices Amer. Math. Soc. 18 (1971), 214. Abstract 682-54-33.

8. T. C. Lim and K. K. Tan, Functional compactness and C-compactness, J. London Math. Soc. (2) 9 (1974), 371-377.

9. R. E. Smithson, Multifunctions, Nieuw Arch. Wisk. (3) 30 (1972), 31-53.

10. G. Viglino, C-compact spaces, Duke Math. J. 36 (1969), 761-764. 
11. __ Seminormal and C-compact spaces, Duke Math. J. 38 (1971), 57-61.

12. A. Bella and F. Cammaroto, On the cardinality of Urysohn spaces, preprint.

13. S. Dolecki and Lechicki, On the structure of upper semicontinuity, J. Math. Anal. Appl. 88 (1982), 547-554.

14. S. Dolecki and S. Rolewicz, Metric characterizations of upper semicontinuity, J. Math. Anal. Appl. 69 (1979), 146-152.

Dipartimento di matematica, Città Universitaria, Viale A. Doria 6, 95125 Catania, Italy 